Proyecciones

Vol. 19, $\mathrm{N}^{\circ}$ 1, pp. 43-52, May 2000

Universidad Católica del Norte

Antofagasta - Chile

\title{
NONEXISTENCE OF NONTRIVIAL SOLUTIONS FOR AN ASYMMETRIC PROBLEM WITH WEIGHTS
}

\author{
A.ANANE and A.DAKKAK \\ Université Moulay Ismail, Marruecos
}

\begin{abstract}
In this paper we establish a nonexistence result for an elliptic problem involving the one-dimentional p-Laplacian operator with asymmetric second member of the equation.
\end{abstract}




\section{Introduction}

Let us to consider the one-dimentional asymmetric problem

$$
\left.-\Delta_{p} u=m_{1}(x) u_{+}^{p-1}-m_{2}(x) u_{-}^{p-1} \text { in }\right] 0, T[,
$$

where $1<p<\infty,\left(m_{1}, m_{2}\right) \in\left(L^{\infty}\right] 0, \mathrm{~T}[)^{2}, u_{ \pm}=\max ( \pm u, 0)$, and $\Delta_{p} u=\left(\left|u^{\prime}\right|^{p-2} u^{\prime}\right)^{\prime}$ denotes the one-dimentional $p$-Laplacian operator

In this paper we study the nonexistence of nontrivial solutions for (1.1) when the pair $\left(m_{1}, m_{2}\right)$ is in some appropriate sense "between two consicutive curves of the Fucik spectrum"; we investigate three situations: The Dirichlet, Neumann and periodic boundary conditions. We recall that the Fucik spectrum for the corresponding boundary conditions wich is noted by $\Theta_{D}\left(\operatorname{resp} . \Theta_{N e}\right)$, (resp. $\left.\Theta_{p e}\right)$ is defined as the set of those $(\mu, \nu) \in \mathbf{R}^{2}$ such that the problem

$$
\left.-\Delta_{p} u=\mu u_{+}^{p-1}-\nu u_{-}^{p-1} \text { in }\right] 0, \mathrm{~T}[
$$

under Dirichlet (resp. Neumann) (resp. periodic) boundary conditions has a nontrivial solution. Several works have been devoted to the study of this spectrum and its applications especially in the higher dimension, cf. [3], [4], [5]. Of particular interest for our purposes are those of P.Drábek [4] where $\Theta_{D}$ is competely determined.

In order to prove our results we use some arguments like the property of the nodal set for eigenfunctions and the simplicity of the first eigenvalue $\lambda_{1}$ of the $p$-Laplacian on $W_{0}^{1, p}$ (cf.[1]) . We will give more details in the next section.

According to the particular quasilinear case where $m_{1}=m_{2}$, one should refer to the interesting work of Del Pino, El Gueta and Manasevich [6], the authors establish a similar result by using the Sturm's comparaison theorem. I suggest that our envisagement is completely different from their approach. Finally I beleive the related result for periodic case is contained in [7], and one should make an appropriate comparaison. 


\section{Statements and proofs.}

\subsection{Curves of the Fucik spectrum and Dirichlet problem.}

In this subsection we shall consider the problems (1.1) and (1.2) under the Dirichlet bounary conditions $u(0)=u(T)=0$. We start by giving the result of P.Drábek [4] on the one-dimentional Fucik spectrum of the $p$-Laplacian on $W_{0}^{1, p}(] 0, \mathrm{~T}[)$. According to the definition of $\Theta_{D}$ given in the introduction, directly we have $\Theta_{D}=\mathbf{R} \times\left\{\lambda_{1}\right\} \cup\left\{\lambda_{1}\right\} \times \mathbf{R}$ $\cup\left(\cup_{n=1}^{\infty}\left[C_{2 n} \cup C_{2 n+1}^{-} \cup C_{2 n+1}^{+}\right]\right)$; where

$$
\begin{aligned}
& C_{2 n}=\left\{(\mu, \nu) \in \mathbf{R}_{+}^{* 2} / n\left[\left(\frac{\lambda_{1}}{\mu}\right)^{1 / p}+\left(\frac{\lambda_{1}}{\nu}\right)^{1 / p}\right]=1\right\} \\
& C_{2 n+1}^{-}=\left\{\begin{array}{r}
(\mu, \nu) \in \mathbf{R}_{+}^{* 2} / n\left[\left(\frac{\lambda_{1}}{\mu}\right)^{1 / p}+\left(\frac{\lambda_{1}}{\nu}\right)^{1 / p}\right]+ \\
+\min \left(\left(\frac{\lambda_{1}}{\mu}\right)^{1 / p},\left(\frac{\lambda_{1}}{\nu}\right)^{1 / p}\right)=1
\end{array}\right\} \\
& C_{2 n+1}^{+}=\left\{\begin{array}{r}
(\mu, \nu) \in \mathbf{R}_{+}^{* 2} / n\left[\left(\frac{\lambda_{1}}{\mu}\right)^{1 / p}+\left(\frac{\lambda_{1}}{\nu}\right)^{1 / p}\right]+ \\
+\max \left(\left(\frac{\lambda_{1}}{\mu}\right)^{1 / p},\left(\frac{\lambda_{1}}{\nu}\right)^{1 / p}\right)=1
\end{array}\right\}
\end{aligned}
$$

Where $\lambda_{1}$ represent the first eigenvalue of the $p$-Laplacian on $W_{0}^{1, p}(] 0, \mathrm{~T}[)$. If we represent $C_{2 n}, C_{2 n+1}^{-}, C_{2 n+1}^{+}$as the curves $\nu=f(\mu)$ so we have the following comparaison:

$$
C_{2 n}<C_{2 n+1}^{-} \leq C_{2 n+1}^{+}<C_{2 n+2} .
$$

Theorem 2.1. Assume that $m_{1}, m_{2} \in L^{\infty}(] 0, T[)$ and there exists $\left(\mu_{1}, \nu_{1}\right),\left(\mu_{2}, \nu_{2}\right) \in \mathbf{R}^{2}$ such that $\left(\mu_{1}, \nu_{1}\right) \lesseqgtr\left(m_{1}(x), m_{2}(x)\right) \lesseqgtr$ $\left(\mu_{2}, \nu_{2}\right)$. Then no nontrivial solutions for (1.1) exists, if one of the following asymptions is true:

1. $\mu_{1}=\nu_{1}=\lambda_{1}$, and $\left(\mu_{2}, \nu_{2}\right) \in C_{2}$.

2. $\left(\mu_{1}, \nu_{1}\right) \in C_{2 n}$, and $\left(\mu_{2}, \nu_{2}\right) \in C_{2 n+1}^{-}$.

3. $\left(\mu_{1}, \nu_{1}\right) \in C_{2 n+1}^{-} \cap \Delta^{ \pm}$, and $\left(\mu_{2}, \nu_{2}\right) \in C_{2 n+1}^{+} \cap \Delta^{ \pm}$; where $\Delta^{+}\left(\right.$resp. $\left.\Delta^{-}\right)=\left\{(\mu, \nu) \in \mathbf{R}^{2} / \mu<\nu(\right.$ resp. $\left.\mu>\nu)\right\}$. 
4. $\left(\mu_{1}, \nu_{1}\right) \in C_{2 n+1}^{+}$, and $\left(\mu_{2}, \nu_{2}\right) \in C_{2 n+2}$.

Remark 2.1. We define the quasilinear problem associated to (1.1) by substituting $m_{1}$ and $m_{2}$ in (1.1) by $m$, so (1.1) is wraten as

$$
\left.-\Delta_{p} u=m(x)|u|^{p-2} u \quad \text { in }\right] 0, T[.
$$

In this particular case the result of [6] remain included in theorem 2.1 .

Proof of theorem 2.1. Suppose that there exists $u \neq 0$ solution of (1.1). We apply Anane's $L^{\infty}$ estimation [2] and Tolksdorf's regularity [8] we have $u \in C^{1}(] 0, T[)$; and by using the maximum principle of Vasquez [9] it easy to see that if $u(a)=0$ then $u^{\prime}(a) \neq 0$, then we deduce $Z(u)=\{x \in[0, \mathrm{~T}] / u(x)=0\}$ is a finite subset. We take

$$
Z(u)=\left\{x_{0}=0<x_{1}<\ldots<x_{k}=\mathrm{T}\right\} .
$$

Put $u_{i}=u /\left[x_{i}, x_{i+1}\right], m_{j, i}=m_{j} /_{\left[x_{i}, x_{i}+1\right]}$ for $j=1,2$ and $i=$ $0, \ldots, k-1$. Since $u$ is a solution of (1.1) under the Dirichlet boundary conditions then $u_{i}$ is a nontrivial solution for some eigenvalue problem with weight like (1.3) with some simple modifications, and by definition $u_{i}$ does not changing sign, then from this fact we have by using the simplicity of $\lambda_{1}$ :

$\lambda_{1}\left(m_{1, i}\right)=1$ or $\lambda_{1}\left(m_{2, i}\right)=1, \forall i=0, \ldots, k-1$. More pricisely, if $u /] x_{0}, x_{1}[>0$ (this hypothesis will be assumed in all the rest of the proof; the similar argument could be adapted to the case $u /] x_{0}, x_{1}[<0$ ); then

$$
\lambda_{1}\left(m_{1,2 q}\right)=1 \quad \text { and } \quad \lambda_{1}\left(m_{2,2 q+1}\right)=1, \ldots \forall q \geq 0 .
$$

by using $\left(\mu_{1}, \nu_{1}\right) \leq\left(m_{1, i}(x), m_{2, i}(x)\right) \leq\left(\mu_{2}, \nu_{2}\right)$ a.e $\left.x \in\right] 0, \mathrm{~T}[\forall i$; the fact that there exists $i_{0}, i_{1}$ such that $\left(\mu_{1}, \nu_{1}\right)<\left(m_{1, i_{0}}(x), m_{2, i_{0}}(x)\right)$ and $\left(m_{1, i_{1}}(x), m_{2, i_{1}}(x)\right)<\left(\mu_{2}, \nu_{2}\right)$ on some subsets of positive measures; and the monotonicity de $\lambda_{1}$ we get: 


$$
\begin{aligned}
& \lambda_{1}\left(\mu_{2} / x_{2 q}, x_{2 q+1}[) \underset{1 \mathrm{st}}{\leq} \lambda_{1}\left(m_{1,2 q}\right)=1 \underset{2 \mathrm{nd}}{\leq} \lambda_{1}\left(\mu_{1} /\right] x_{2 q}, x_{2 q+1}[)\right. \\
& \lambda_{1}\left(\nu_{2} /\right] x_{2 q+1}, x_{2 q+2}[) \underset{1 \mathrm{st}}{\leq} \lambda_{1}\left(m_{2,2 q+1}\right)=1 \underset{2 \mathrm{nd}}{\leq} \lambda_{1}\left(\nu_{1} /\right] x_{2 q+1}, x_{2 q+2}[),
\end{aligned}
$$

and there exists some integers $q$ such that one of two last firsts (1st) and one of two last seconds (2nd) inequalitys was stricts.

From [4] and for some positive constant $\alpha$ we get,

$$
\left.\lambda_{1}(\alpha /]_{a, b[}\right)=\frac{1}{\alpha}\left(\frac{\pi_{p}}{b-a}\right)^{p} ; \pi_{p}=2(p-1)^{p} \int_{0}^{1} \frac{d s}{\left(1-s^{p}\right)^{1 / p}},
$$

then by using the last inequalitys we obtain :

$\frac{\pi_{p}}{\left(\mu_{2}\right)^{1 / p}} \underset{1 \mathrm{st}}{\leq} x_{2 q+1}-x_{2 q} \underset{\text { 2nd }}{\leq} \frac{\pi_{p}}{\left(\mu_{1}\right)^{1 / p}}, \quad \frac{\pi_{p}}{\left(\nu_{2}\right)^{1 / p}} \underset{1 \mathrm{st}}{\leq} x_{2 q+2}-x_{2 q+1} \underset{2 \mathrm{nd}}{\leq} \frac{\pi_{p}}{\left(\nu_{1}\right)^{1 / p}}$,

and one of those 1st and one of those 2nd last inequalitys was stricts, then by according to this point and by sommons in the last inequalitys from $q=0$ to $\frac{k-1}{2}$ we give

$$
\begin{aligned}
E\left(\frac{k}{2}\right)\left[\frac{\pi_{p}}{\left(\mu_{2}\right)^{1 / p}}+\frac{\pi_{p}}{\left(\nu_{2}\right)^{1 / p}}\right]+\varepsilon \frac{\pi_{p}}{\left(\mu_{2}\right)^{1 / p}}<T<E\left(\frac{k}{2}\right)\left[\frac{\pi_{p}}{\left(\mu_{1}\right)^{1 / p}}+\frac{\pi_{p}}{\left(\nu_{1}\right)^{1 / p}}\right]+ \\
+\varepsilon \frac{\pi_{p}}{\left(\mu_{1}\right)^{1 / p}},
\end{aligned}
$$

where $\varepsilon=0$ if $k$ is even and $\varepsilon=1$ if not. So we obtain:

$$
\begin{aligned}
E\left(\frac{k}{2}\right)\left[\left(\frac{\lambda_{1}}{\mu_{2}}\right)^{1 / p}+\left(\frac{\lambda_{1}}{\nu_{2}}\right)^{1 / p}\right]+\varepsilon\left(\frac{\lambda_{1}}{\mu_{2}}\right)^{1 / p}<1<E\left(\frac{k}{2}\right)\left[\left(\frac{\lambda_{1}}{\mu_{1}}\right)^{1 / p}\right. & \left.+\left(\frac{\lambda_{1}}{\nu_{1}}\right)^{1 / p}\right]+ \\
& +\varepsilon\left(\frac{\lambda_{1}}{\mu 1}\right)^{1 / p} .
\end{aligned}
$$

By using the hypothesis 2) (for exemple) of therem 2.1; the definition of $C_{2 n}, C_{2 n+1}^{-}$and the last inequalitys it was easy to see that $n<E\left(\frac{k}{2}\right)<n+1$; wich gives a contradiction. The same conclusion will be established if we consider the hypothesis 1) or 3) or 4) of theorem 2.1. 


\subsection{Neumann Problem.}

In this subsection we shall consider the problems (1.1) and (1.2) under the Neumann bounary conditions $u^{\prime}(0)=u^{\prime}(T)=0$.

Theorem 2.2. Assume that $m_{1}, m_{2} \in L^{\infty}(] 0, T[)$ and there exists $\left(\mu_{1}, \nu_{1}\right),\left(\mu_{2}, \nu_{2}\right) \in \mathbf{R}^{2}$ such that $\left(\mu_{1}, \nu_{1}\right) \underset{\ddagger}{\lessgtr}\left(m_{1}(x), m_{2}(x)\right) \underset{\ddagger}{\lessgtr}$ $\left(\mu_{2}, \nu_{2}\right)$. Then no nontrivial solutions for (1.1) exists, if one of the following asymptions is true:

1. $\mu_{1}=\nu_{1}=0$, and $\left(\mu_{2}, \nu_{2}\right) \in \bar{C}_{1}$.

2. $\left(\mu_{1}, \nu_{1}\right) \in \bar{C}_{n}$, and $\left(\mu_{2}, \nu_{2}\right) \in \bar{C}_{n+1} \quad(n \geq 1)$.

Where $\bar{C}_{n}=\left\{(\mu, \nu) \in \mathbf{R}_{+}^{* 2} / n\left[\left(\frac{\lambda_{1}}{\mu}\right)^{1 / p}+\left(\frac{\lambda_{1}}{\nu}\right)^{1 / p}\right]=2\right\}$.

Proof. Suppose that there exists $u \neq 0$ solution de (1.1). Then by according to the beginning of the proof of theorem 2.1 we get $u(0) \neq 0$, $u(T) \neq 0$ and $Z(u)$ is a finite subset. Let $Z(u)=\left\{x_{1}<\ldots<x_{k}\right\}$. We define

$$
\begin{gathered}
\bar{u}(x)=u(-x) \quad \text { if }-x_{1} \leq x \leq 0, \\
\bar{u}(x)=u(x) \quad \text { if } 0 \leq x \leq \mathrm{T}, \\
\bar{u}(x)=u(2 \mathrm{~T}-x) \text { if } \mathrm{T} \leq x \leq 2 \mathrm{~T}-x_{k}
\end{gathered}
$$

and the analogous definition for $\bar{m}_{1}$ and $\bar{m}_{2}$. It easy to see that $\bar{u}$ is a solution of a Dirichlet problem like (1.3) with domain ] $-x_{1}, 2 \mathrm{~T}-x_{k}$ [ and weights $\left(\bar{m}_{1}, \bar{m}_{2}\right)$.

Without loss of generaliy we can assume that $u /{ }_{0}, x_{1}[>0$; and in order to simplify this proof we shall us simply the following hypothesis (of therem 2.2):

$$
\left.\mu_{1}<m_{1}(x)<\mu_{2} \quad \text { and } \quad \nu_{1}<m_{1}(x)<\nu_{2} \quad \text { a.e } x \in\right] 0, \mathrm{~T}[.
$$

We proceed as in the proof of theorem 2.1, then we obtain:

1. $\frac{\pi_{p}}{\left(\mu_{2}\right)^{1 / p}}<2 x_{1}<\frac{\pi_{p}}{\left(\mu_{1}\right)^{1 / p}}$ 
2. $\frac{\pi_{p}}{\left(\mu_{2}\right)^{1 / p}}<x_{2}-x_{1}<\frac{\pi_{p}}{\left(\mu_{1}\right)^{1 / p}} \quad \cdots$ k. $\frac{\pi_{p}}{\left(\nu_{2}\right)^{1 / p}}<x_{\mathrm{k}}-x_{\mathrm{k}-1}<\frac{\pi_{p}}{\left(\nu_{1}\right)^{1 / p}}$ if $k$ is even ; $\frac{\pi_{p}}{\left(\mu_{2}\right)^{1 / p}}<x_{\mathrm{k}}-x_{\mathrm{k}-1}<\frac{\pi_{p}}{\left(\mu_{1}\right)^{1 / p}}$
if not

$\mathrm{k}+1 . \frac{\pi_{p}}{\left(\mu_{2}\right)^{1 / p}}<2 \mathrm{~T}-2 x_{\mathrm{k}}<\frac{\pi_{p}}{\left(\mu_{1}\right)^{1 / p}}$ if $k$ is even $; \frac{\pi_{p}}{\left(\nu_{2}\right)^{1 / p}}<2 \mathrm{~T}-2 x_{\mathrm{k}}<$ $\frac{\pi_{p}}{\left(\nu_{1}\right)^{1 / p}}$ if not.

We multiply the equations 2 to $k$ by 2 and we sum from 1 to $k+1$, wich gives:

$$
k\left[\frac{\pi_{p}}{\left(\mu_{2}\right)^{1 / p}}+\frac{\pi_{p}}{\left(\nu_{2}\right)^{1 / p}}\right]<2 T<k\left[\frac{\pi_{p}}{\left(\mu_{1}\right)^{1 / p}}+\frac{\pi_{p}}{\left(\nu_{1}\right)^{1 / p}}\right],
$$

SO

$$
k\left[\left(\frac{\lambda_{1}}{\mu_{2}}\right)^{1 / p}+\left(\frac{\lambda_{1}}{\nu_{2}}\right)^{1 / p}\right]<2<k\left[\left(\frac{\lambda_{1}}{\mu_{1}}\right)^{1 / p}+\left(\frac{\lambda_{1}}{\nu_{1}}\right)^{1 / p}\right]
$$

since $\left(\mu_{1}, \nu_{1}\right) \in \bar{C}_{n}$, and $\left(\mu_{2}, \nu_{2}\right) \in \bar{C}_{n+1}$ then it was easy to see that $n<k<n+1$, wich gives a contradiction.

Corollary 2.1. The one-dimensional Fucik spectrum for the $p$ Laplacian with Neumann boundary conditions is: $\Theta_{\mathrm{Ne}}=\mathbf{R} \times\{0\} \cup$ $\{0\} \times \mathbf{R} \cup\left(\cup_{n=1}^{\infty} \bar{C}_{n}\right)$.

So we deduce the usuel one-dimensional spectrum for the $p$-Laplacian with Neumann boundary conditions is given by :

$$
\sigma_{\mathrm{Ne}}\left(-\Delta_{p}\right)=\left\{n^{p} \lambda_{1} / n \geq 0\right\}
$$

Remark 2.2. If $(\mu, \nu) \in \bar{C}_{1}$ then $\lim _{\mu \rightarrow+\infty} \nu(\mu)=\frac{\lambda_{1}}{2^{p}}>0$; (here 0 represent the first eigenvalue of the $p$-Laplacian with Neumann boundary conditions). In [3] the authors show that there exists a connection between this asymptotic behaviour of $\bar{C}_{1}$ and the antimaximum principle; but if the dimension is larger then 2 and $p=2$ so we have $\bar{C}_{1}$ is asymptotic to 0 (cf.[5]). In the Dirichlet case the first curve of the Fucik spectrum is always asymptotic to $\lambda_{1}$. 


\subsection{Periodic Problem.}

In this subsection we shall consider the problems (1.1) and (1.2) under periodic bounary conditions: $u(0)-u(T)=u^{\prime}(0)-u^{\prime}(T)=0$.

Theorem 2.3. Assume that $m_{1}, m_{2} \in L^{\infty}(] 0, T[)$ and there exists $\left(\mu_{1}, \nu_{1}\right),\left(\mu_{2}, \nu_{2}\right) \in \mathbf{R}^{2}$ such that $\left(\mu_{1}, \nu_{1}\right) \underset{\ddagger}{\lessgtr}\left(m_{1}(x), m_{2}(x)\right) \underset{\ddagger}{\lessgtr}$ $\left(\mu_{2}, \nu_{2}\right)$ a.e $\left.x \in\right] 0, \mathrm{~T}[$. Then no nontrivial solutions for (1.1) exists, if one of the following asymptions is true:

1. $\mu_{1}=\nu_{1}=0$, and $\left(\mu_{2}, \nu_{2}\right) \in \widetilde{C}_{1}$.

2. $\left(\mu_{1}, \nu_{1}\right) \in \widetilde{C}_{n}$, and $\left(\mu_{2}, \nu_{2}\right) \in \widetilde{C}_{n+1} \quad(n \geq 1)$.

Where $\widetilde{C}_{n}=\left\{(\mu, \nu) \in \mathbf{R}_{+}^{* 2} / n\left[\left(\frac{\lambda_{1}}{\mu}\right)^{1 / p}+\left(\frac{\lambda_{1}}{\nu}\right)^{1 / p}\right]=1\right\}$.

Proof. Suppose that there exists $u \neq 0$ solution of (1.1) . Put $Z(u)=\left\{x_{1}<\ldots<x_{k}\right\}$. We define:

$\widetilde{u}(x)=u(x+\mathrm{T}) \quad$ if $x_{k}-\mathrm{T} \leq x \leq 0$,

$\widetilde{u}(x)=u(x) \quad$ if $0 \leq x \leq \mathrm{T}$,

$\widetilde{u}(x)=u(2 \mathrm{~T}-x) \quad$ if $\mathrm{T} \leq x \leq \mathrm{T}+x_{1}$

and the analogous definition for $\widetilde{m}_{1}$ and $\widetilde{m}_{2}$. It easy to see that $\widetilde{u}$ is a nontrivial solution for a Dirichlet problelm like (1.3) with domain ]$x_{k}-\mathrm{T}, \mathrm{T}+x_{1}\left[\right.$ and weights $\left(\widetilde{m}_{1}, \widetilde{m}_{2}\right)$.

In order to simplify this proof we shall assume

$0<x_{1}<x_{k}<\mathrm{T},\left(\mu_{1}, \nu_{1}\right)<\left(m_{1}(x), m_{2}(x)\right)<\left(\mu_{2}, \nu_{2}\right)$ a.e, and $u /] 0, x_{1}[>0$.

In the fact that $u^{\prime}(0)-u^{\prime}(T)=0$ we obtain that $k$ is even, so by a similar arguments of the proof of theorem 2.2 and by using the hypothesis 2) of theorem 2.3 we get:

1. $\frac{\pi_{p}}{\left(\mu_{2}\right)^{1 / p}}<\mathrm{T}+x_{1}-x_{\mathrm{k}}<\frac{\pi_{p}}{\left(\mu_{1}\right)^{1 / p}}$ 


$$
\begin{aligned}
\text { 2. } & \frac{\pi_{p}}{\left(\mu_{2}\right)^{1 / p}}<x_{2}-x_{1}<\frac{\pi_{p}}{\left(\mu_{1}\right)^{1 / p}} \quad \cdots \\
\text { k. } & \frac{\pi_{p}}{\left(\nu_{2}\right)^{1 / p}}<x_{\mathrm{k}}-x_{\mathrm{k}-1}<\frac{\pi_{p}}{\left(\nu_{1}\right)^{1 / p}} \\
\mathrm{k}+1 . & \frac{\pi_{p}}{\left(\mu_{2}\right)^{1 / p}}<\mathrm{T}+x_{1}-x_{\mathrm{k}}<\frac{\pi_{p}}{\left(\mu_{1}\right)^{1 / p}}
\end{aligned}
$$

we multiply the equations 2 to $k$ by 2 and we sum from 1 to $k+1$, we have

$$
\frac{k}{2}\left[\left(\frac{\lambda_{1}}{\mu_{2}}\right)^{1 / p}+\left(\frac{\lambda_{1}}{\nu_{2}}\right)^{1 / p}\right]<1<\frac{k}{2}\left[\left(\frac{\lambda_{1}}{\mu_{1}}\right)^{1 / p}+\left(\frac{\lambda_{1}}{\nu_{1}}\right)^{1 / p}\right]
$$

Since $\left(\mu_{1}, \nu_{1}\right) \in \widetilde{C}_{n}$, and $\left(\mu_{2}, \nu_{2}\right) \in \widetilde{C}_{n+1}$ then it easy to see that $n<\frac{k}{2}<n+1$ wich gives a contradiction because $k$ is even.

Corollary 2.2. The one-dimensional Fucik spectrum for the $p$ Laplacian with periodic boundary conditions is: $\Theta_{\text {pe }}=\mathbf{R} \times\{0\} \cup$ $\{0\} \times \mathbf{R} \cup\left(\cup_{n=1}^{\infty} \widetilde{C}_{n}\right)$.

So we deduce the usuel one-dimensional spectrum for the $p$-Laplacian with periodic boundary conditions is given by :

$$
\sigma_{\mathrm{pe}}\left(-\Delta_{p}\right)=\left\{(2 n)^{p} \lambda_{1} / n \geq 0\right\} \text {. }
$$

\section{REFERENCES.}

[1] A. Anane, Simplicité et isolation de la première valeure propre du p-laplacien avec poids, C. R. Acad. Sci. Paris, t. 305, pp 725-728, (1987).

[2] A. Anane, Etude des valeurs propres et de la résonance pour l'opérateur p-laplacien, thèse de Doctorat, Université Libre de Bruxelles, (1988).

[3] M. Arias, J. Campos \& J.-P. Gossez, On the antimaximum principle and the Fucik spectrum for the Neumann $p$-Laplacien, (to appear in Diff. Int. Equa.). 
[4] P. Drábek. Solvability and bifurcations of nonlinear equations, Pitman Resarch Notes in Mathematics, 264 (1992).

[5] D. G. de Figueredo \& J.-P. Gossez, On the first curve of the Fucik spectrum of an elliptic operator, Diff. Int. Equa., volume 7, number 5, pp-1285-1302, (1994).

[6] M. del Pino, M. Elgueta \& R. Manasevich, A homotopic deformation along $p$ of a Leray-Shauder degree result and existence for $\left(\left|u^{\prime}\right|^{p-2} u^{\prime}\right)^{\prime}+f(t, u)=0, u(0)=u(T)=0, p>1$. J. Diff. Eq. , 80, pp 1-13, (1989).

[7] Del Pino, Manasevich \& Murua. ....,,Nonlinear Analysis 18, pp 79-92, (1992)

[8] P. Tolksdorf, Regularity for more general class of quasilinear elliptic equation, J. Diff. Eq. , 8, pp 773-817, (1983).

[9] J. L.Vasquez, A strong maximum principle for quasilinear equations, Appl. Math. Optim., 12, pp 191-202, (1984).

Received : October 10, 1999.

\author{
A. Anane \\ and \\ Ahmed Dakkak \\ Département de Mathématiques \\ Faculté des Sciences et Techniques \\ Université Moulay Ismail \\ B. P. 509 Boutalamine \\ Errachidia \\ Maroc
}

